\title{
NOUVELL
}

\section{À quoi pensent les singes quand ils cherchent leur nourriture?}

\section{Une approche comparative à l'interface des neurosciences et de l'écologie comportementale}

Sébastien Bouret ${ }^{1}$, Margot Louail ${ }^{1}$, Sandrine Prat ${ }^{2}$, Cécile Garcia ${ }^{3}$

$>$ Comment pensent les animaux? Pour tenter de répondre à cette question, les chercheurs utilisent des mesures indirectes, comme l'étude du comportement ou des caractéristiques morphologiques du cerveau, notamment sa taille. Chez les primates, l'hypothèse du 'cerveau social' prédit que la pensée est d'autant plus riche et complexe que les individus vivent au sein de groupes sociaux importants, en postulant que les interactions sociales impliquent une manipulation complexe des représentations de la pensée et des intentions des autres individus du groupe. Cette théorie s'appuie sur des analyses qui montrent une corrélation positive entre la taille du cerveau et la taille du groupe social chez les primates [1,2]. Mais d'autres théories ont été proposées pour expliquer le développement des capacités mentales des primates. Selon l'hypothèse du «cerveau écologique » $[3,4]$, les capacités mentales seraient liées à la recherche de nourriture: les paramètres écologiques liés à celle-ci auraient favorisé l'évolution de la taille du cerveau. Ainsi, les animaux dont le régime alimentaire est simple et repose sur des ressources largement disponibles auraient moins besoin de représentations mentales pour se nourrir, tandis que ceux pour lesquels la nourriture est variable dans le temps et dans l'espace, ou difficile à obtenir, auraient développé des capacités mentales plus importantes. Cette théorie a récemment reçu l'appui d'une étude montrant que la taille du cerveau des primates était plus étroitement reliée à la richesse du régime alimentaire qu'à la taille du groupe [5]. En effet, les primates frugivores ont un cerveau plus développé que les primates exclusivement folivores: pour trouver des fruits, il faut être capable de se souvenir de la position des arbres producteurs selon les
${ }^{1}$ CNRS - Association Sorbonne Université, Musée de l'Homme, 17 place du Trocadéro, 75016 Paris, France.

2UMR 7194 (HNHP), MNHN/CNRS/UPVD, Association Sorbonne Université, Musée de I'Homme, 17 place du Trocadéro, 75016 Paris, France.

${ }^{3}$ UMR 7206 Éco-anthropologie, CNRS -

MNHN - Université de Paris,

Musée de l'Homme,

17 place du Trocadéro, 75016 Paris, France. sebastien.bouret@hotmail.com

saisons, de leur niveau de fructification, et d'organiser les déplacements correspondants [6]. Chez les primates folivores, la recherche des feuilles constitue un défi moindre puisque celles-ci sont généralement abondantes et faciles à trouver. La pression écologique sur le développement des capacités mentales est donc plus faible chez les espèces folivores, ce qui rendrait compte du fait que leur cerveau est plus petit. Mais que signifie cette différence de taille du cerveau? On peut penser que l'organisation globale du cerveau des primates est suffisamment homogène entre espèces pour que l'on puisse interpréter les différences de taille sur le plan quantitatif, plutôt que qualitatif. En d'autres termes, les opérations mentales seraient de même nature, mais elles seraient 


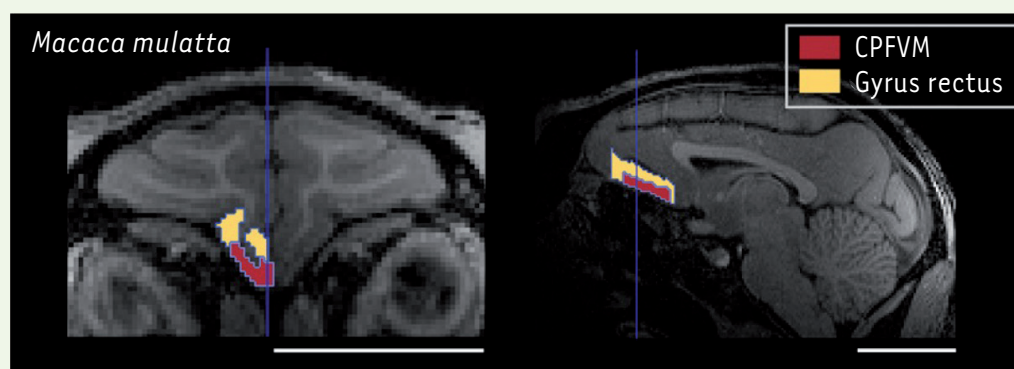

Macaca fuscata
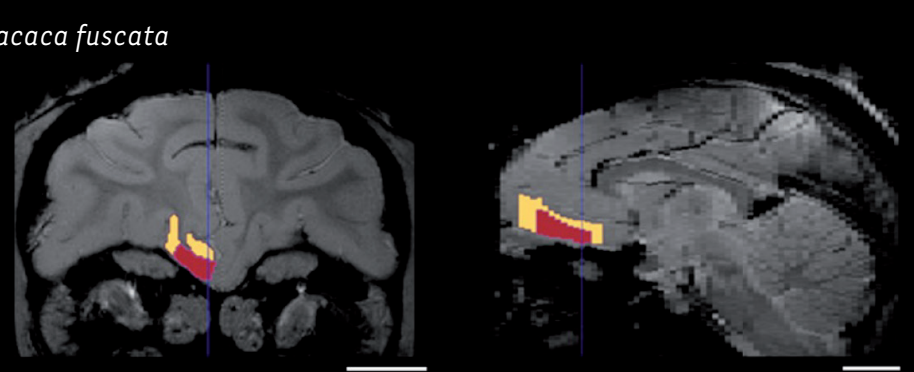

Gorilla gorilla
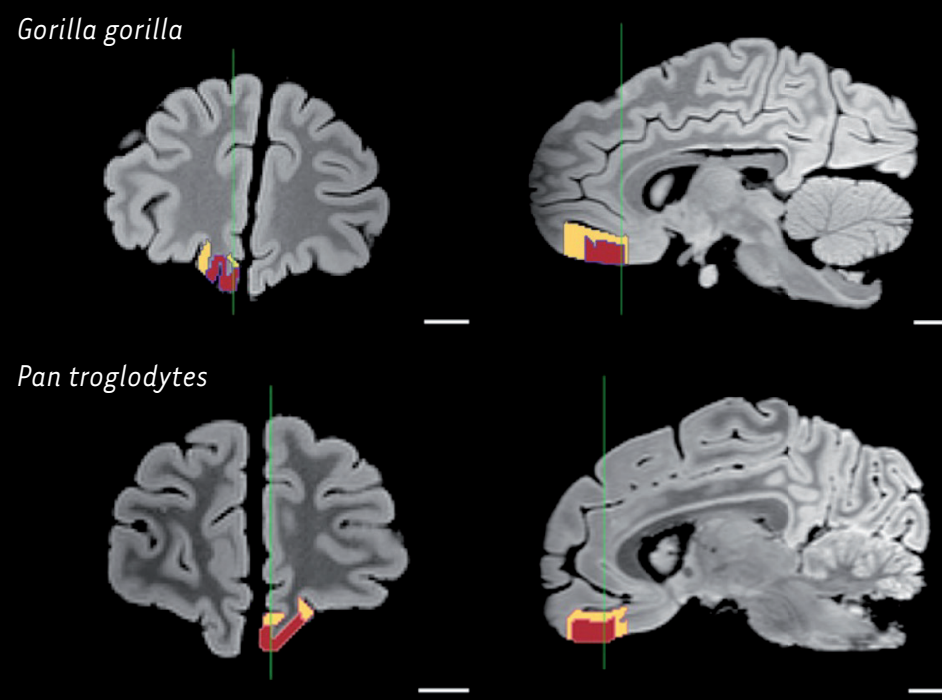

Homo sapiens
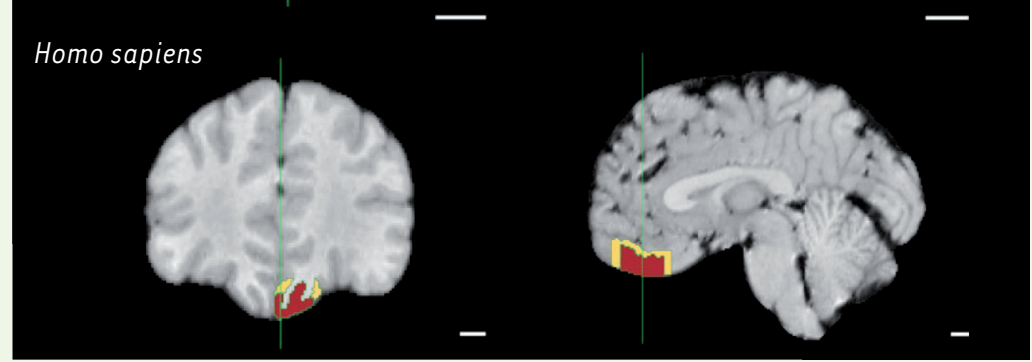

Figure 1. Délimitation des régions cérébrales d'intérêt dans cinq espèces de primates. Ces régions ont été délimitées en utilisant des images de cerveau obtenues par imagerie par résonance magnétique. Vues en coupes coronales (à gauche) et sagittales (à droite) du cerveau d'un individu représentatif de chaque espèce. Deux régions sont délimitées: le cortex préfrontal ventro-médian (CPFVM, en rouge) et le gyrus rectus (en jaune). Le CPFVM est la région d'intérêt, impliquée dans la prise de décision et la mémoire. Le gyrus rectus est une circonvolution bien visible, qui inclut le CPFVM, et qui a servi de référence. La diversité du régime alimentaire entre ces espèces ne permet d'expliquer que la taille relative du CPFVM, mais pas celle du gyrus rectus.

plus ou moins développées. L’hypothèse alternative serait que certaines espèces développent des capacités cognitives que d'autres n'ont pas: les différences seraient alors qualitatives.

Les neurosciences cognitives peuvent apporter des réponses à ces questions. Depuis l'avènement de la neuropsychologie, nous savons qu'il existe une relation spécifique entre certaines opérations mentales et certaines régions du cerveau. Par exemple, les processus de production de la parole et de compréhension du langage dans l'espèce humaine mettent en jeu différentes aires cérébrales, i.e. une région du lobe frontal gauche (l'aire de Broca) et une région du lobe temporal gauche (l'aire de Wernicke). De même, une autre région du lobe temporal, l'hippocampe, joue un rôle important dans la représentation de l'espace. Or il a été montré que cette région était en moyenne plus développée chez les personnes qui ont souvent recours à des représentations mentales de leur environnement pour se déplacer (par exemple, les chauffeurs de taxi) [7]. On trouve également cette relation entre la taille d'une région spécifique du cerveau et la mobilisation de sa fonction supposée dans le domaine des interactions sociales [8]. II a également été montré que la prise de décision fondée sur la valeur des options dépend d'une région précise du lobe frontal, le cortex préfrontal ventro-médian (CPFVM) $[9,10]$. Ces études, effectuées en laboratoire, montrent à quel point les relations entre certaines régions du cerveau et le développement de fonctions cognitives définies sont spécifiques. Ainsi, I'hypothèse du «cerveau écologique » et celle du «cerveau social » ne sont pas en opposition puisque les fonctions associées dépendraient de régions cérébrales différentes. Cependant, la cartographie cérébrale de ces fonctions ayant été établie en laboratoire, on peut se demander à quel point les régions identifiées et leurs fonctions associées sont pertinentes pour comprendre le comportement des animaux dans la nature. En d'autres termes, peut-on relier la taille 
d'une région cérébrale donnée, associée à une opération cognitive spécifique, à une fonction écologique pertinente pour les animaux dans leur milieu naturel ?

Pour répondre à cette question, nous avons mis en œuvre une approche pluridisciplinaire, combinant écologie comportementale et neurosciences cognitives, qui permet d'utiliser conjointement les forces de chacune de ces disciplines pour dépasser les limites des autres [11]. Dans le cadre théorique de l'hypothèse du «cerveau écologique », nous avons testé l'hypothèse selon laquelle la taille du CPFVM (région impliquée dans la prise de décision fondée sur la valeur, et donc vraisemblablement dans la planification des actions en fonction de la valeur des objectifs) joue un rôle clé dans la recherche de nourriture chez les primates. Selon cette hypothèse, cette région devrait être plus développée chez les espèces ayant un régime alimentaire varié que chez celles dont le régime alimentaire présente moins de diversité. Par ailleurs, cette relation devrait être spécifique, et donc ne pas être trouvée dans d'autres régions cérébrales associées à d'autres fonctions. Enfin, la variation de la taille du CPFVM entre espèces devrait être plus étroitement reliée à la recherche de nourriture qu'à d'autres fonctions cérébrales (e.g., interactions sociales). Nous avons mesuré la taille du CPFVM à partir de données de neuroimagerie anatomique dans cinq espèces de primates: Homo sapiens, Macaca mulatta, Macaca fuscata, Gorilla gorilla, et Pan troglodytes (chimpanzé commun) (Figure 1). Notre échantillon incluait entre 5 et 7 individus par espèce. Pour évaluer la spécificité de la relation, nous avons également mesuré la taille du gyrus rectus, une région plus grande du lobe frontal qui inclut le CPFVM, ainsi que la taille du cortex somato-sensoriel, situé à l'avant du lobe pariétal et supposé ne pas être impliqué dans la fonction d'intérêt. Nous avons également mesuré la taille du cerveau entier, afin de faciliter la comparaison avec les études antérieures. En parallèle, nous avons construit une base de données regroupant, pour chaque espèce: 1) des variables écologiques pertinentes pour la recherche de nourriture (e.g., variabilité du régime alimentaire, distance parcourue par jour), 2) des variables plus étroitement reliées aux interactions sociales (e.g., taille du groupe), et 3) la distance phylogénétique avec les autres espèces. Nous avons ensuite construit un ensemble de modèles mathématiques incluant diverses combinaisons de variables socio-écologiques, ainsi que la distance phylogénétique moyenne, pour expliquer la taille de chacune de nos régions d'intérêt. $\varepsilon$ n comparant la puissance statistique de ces modèles, nous avons identifié les combinaisons de variables socio-écologiques qui expliquaient le mieux les différences de taille de chacune des régions d'intérêt chez ces espèces de primates.

Nous avons ainsi montré que la taille du CPFVM est particulièrement corrélée à deux facteurs écologiques liés à l'alimentation: la diversité alimentaire et l'âge de sevrage. Dans les deux cas, le lien entre la taille du CPFVM et le régime alimentaire peut être expliqué par un besoin plus important d'une représentation mentale de la valeur des options (buts) pour la planification des actions. Nous n'avons pas retrouvé cette relation pour le cortex somato-sensoriel, comme attendu, ni pour le gyrus rectus tout entier, ce qui est plus surprenant dans la mesure où cette région inclut le CPFVM. Cependant, nous avons également remarqué que les modèles socioécologiques expliquant la taille du gyrus rectus étaient plus proches de ceux qui expliquent la taille du cerveau entier. Sachant que le lobe frontal, en particulier le cortex préfrontal, est la partie du cerveau qui diffère le plus entre les primates, et que le cortex préfrontal joue un rôle important dans l'ensemble des fonctions exécutives et la planification, la variation de taille du CPFVM que nous avons observée est bien liée à une fonction spécifique, et non à un accroissement global des fonctions exécutives. En conclusion de cette étude, nous suggérons que l'accroissement d'une région spécifique du cerveau, le CPFVM, a permis à certains primates de développer des stratégies de recherche de nourriture complexes et adaptées aux variations saisonnières et spatiales des ressources alimentaires. Reste à comprendre la réorganisation cérébrale et cognitive liée à la recherche de nourriture et à l'utilisation ou la fabrication d'outils au cours de l'évolution de l'espèce humaine. $\diamond$

What do primates think about when foraging? A comparative approach at the interface between neurosciences and behavioural ecology

\section{LIENS D'INTÉRÊT}

Les auteurs déclarent n'avoir aucun lien d'intérêt concernant les données publiées dans cet article.

\section{RÉFÉRENCES}

1. Dunbar RI. The social brain hypothesis. Brain 1998 ; 9 : 178-90.

2. Dunbar $R$, Shultz $S$. Why are there so many explanations for primate brain evolution? Philos Trans R Soc Lond B, Biol Sci 2017 ; 372. doi : 10.1098/ rstb.2016.0244.

3. Milton K. Distribution patterns of tropical plant foods as an evolutionary stimulus to primate mental development. Am Anthropol 1981 ; 83 : 534-48.

4. Milton K. Foraging behaviour and the evolution of primate intelligence. In: Byrne RW, Whiten A, eds. Machiavellian intelligence: social expertise and the evolution of intellect in monkeys, apes, and humans. New York: Clarendon Press/Oxford University Press, 1988: 285-305.

5. DeCasien AR, Williams SA, Higham JP. Primate brain size is predicted by diet but not sociality. Nat $\varepsilon$ col Evol 2017 ; 1 : 112.

6. Janmaat KR, Chapman CA, Meijer R, Zuberbühler K. The use of fruiting synchrony by foraging mangabey monkeys: a "simple tool" to find fruit. Anim Cogn 2011 ; $15: 83-96$.

7. Maguire $\varepsilon A$, Gadian DG, Johnsrude IS, et al. Navigation-related structural change in the hippocampi of taxi drivers. Proc Natl Acad Sci USA $2019 ; 97: 4398-403$.

8. Sallet J, Mars RB, Noonan MP, et al. Social network size affects neural circuits in macaques. Science 2011 ; $334: 697-700$.

9. Bartra 0, McGuire JT, Kable JW. The valuation system: a coordinate-based meta-analysis of BOLD fMRI experiments examining neural correlates of subjective value. Neurolmage 2013 ; 76 : 412-27.

10. Benoit RG, Szpunar KK, Schacter DL. Ventromedial prefrontal cortex supports affective future simulation by integrating distributed knowledge. Proc Natl Acad Sci USA $2014 ; 111$ : 16550-5.

11. Louail M, Gilissen $\varepsilon$, Prat $S$, et al. Refining the ecological brain: strong relation between the ventromedial prefrontal cortex and feeding ecology in five primate species. Cortex 2019 ; 118 : 262-74. 